\title{
UNIDAD ENTRE SENSIBILIDAD Y ENTENDIMIENTO. EL ORIGEN DEL PROBLEMA CRÍTICO ${ }^{12}$
}

\author{
Mario Molina Maydl \\ Instituto de Sociología \\ Pontificia Universidad Católica de Chile
}

\begin{abstract}
Resumen / Abstract
Este artículo se propone mostrar que el surgimiento del problema crítico, entre el periodo pre-crítico de 1768 y 1772, involucra la relación de dos cuestiones: por una parte, el hecho de que espacio y tiempo sean formas puras de objetos sensibles; y por otra, el hecho de que las representaciones intelectuales requieran aplicarse a los objetos sensibles a través de la sensibilidad. Esto requerirá plantear la pregunta por la unidad entre sensibilidad y entendimiento.
\end{abstract}

Palabras Clave: dualismo, sensibilidad, capacidad receptiva, actividad.

THE UNITY BETWEEN SENSIBILITY AND UNDERSTANDING. THE ORIGIN OF THE CRITICAL PROBLEM

This article aims at showing that the emergence of the critical problem, between the pre-critic years 1768 and 1772, involves the relationship of two statements: on the one side, (i) the fact that space and time are pure forms of sensible objects; and on the other, (ii) that intellectual representations claim for being applied to sensible objects through sensibility. This will require to bring up the issue for the unity of sensibility and understanding.

KEY WORDS: dualism, sensibility, receptive capacity, activity.

$\mathbb{R A}$

Para la elaboración de este trabajo, asumo como punto de partida un determinado modo de comprender la problemática existente en la Kritik der reinen Vernunft (desde ahora $\mathrm{KrV}^{3}$ ) en lo que se refiere a la relación entre las dos fuentes fundamentales (Grundquellen) del conocimiento, sensibilidad y entendimiento ${ }^{4}$. Sostengo que estas

\footnotetext{
Una versión preliminar de este trabajo aparece en Molina (2012).

Agradezco los comentarios de José Antonio Giménez y de los profesores Alejandro Vigo y Hugo Herrera, además de las valiosas sugerencias de los correctores. Está demás decir que los errores que contenga el presente artículo son de mi responsabilidad.

3 Refiero a la $\mathrm{KrV}$ en sus dos ediciones (A y B). Las referencias a los demás textos de Kant se hacen según la edición de la Academia $=$ Ak.

4 Cf. $\mathrm{Kr} V, \mathrm{~A} 50 / \mathrm{B} 74$.
} 
dos facultades se encuentran en una relación esencial, es decir, en una relación tal que el ser de una facultad se explica por el ser de la otra, de tal modo que la experiencia de las cosas del mundo requiere de esta precisa conexión para hacer sentido de cómo una representación pueda ser aplicada sobre un objeto, es decir, para comprender cómo es posible que una representación conceptual pueda referir a la existencia de las cosas del mundo.

Por motivos de espacio, no puedo extenderme acá acerca de la naturaleza de este tipo de relación. Pero esta idea debe ser considerada como el hilo conductor de la interpretación de los textos precríticos que me propongo presentar a continuación y que constituye, en definitiva, un modo de rastrear las bases para una presentación del surgimiento del problema crítico. En este sentido, los argumentos presentados obedecerán a una comprensión genético-sistemática del periodo precrítico comprendido entre los años 1768 y 1772 . He decidido considerar esta etapa, porque en 1768 Kant plantea por primera vez, al menos, una preconcepción idealista de la sensibilidad ${ }^{5}$, que me parece ser el punto de arranque del planteamiento del problema crítico que Kant establece por primera vez en la conocida carta dirigida a Marcus Herz en 1772 y que luego resolverá en la presentación de las dos ediciones de la $\mathrm{KrV}$.

Me interesa mostrar en lo que sigue cómo la división explícita de las facultades que Kant realiza en 1770 (y que de hecho separa radicalmente), en el texto De mundi sensibilis atque intelligibilis forma et principiis (Acerca de la forma del mundo sensible e inteligible y sus principios; en adelante Dissertatio), se constituye como el paso

5 Es verdad que en el opúsculo de 1968 (al que referiré más adelante) Kant parece defender la posición del newtoniano Euler en contra de la posición de Leibniz (cf. Vaihinger 1892, vol. 2, pp. 425-6; Torretti 2005, pp. 161-2; Torretti 2004, pp. 112-3). Sin embargo, a mi modo de ver, este texto temprano de Kant $(i)$ no establece ninguna afirmación que sea específicamente atribuible a favor de la posición substancialista del espacio y (ii) la posición que establece no es incompatible con la tesis que mantendrá luego en 1770 (Torretti 2005, pp. 161-2). Respecto de (i), la tesis del opúsculo es mostrar que "el espacio absoluto (der absolute Raum) $<$ es $>$ independiente de la existencia de toda materia y, en cuanto fundamento primero de la posibilidad de su conexión (Zusammensetzung) [el de la materia], tiene una realidad propia (eine eigene Realität)" (Ak. 2:378; traducción mía; énfasis mío). Efectivamente, esta afirmación da pie para pensar que Kant sostiene la posición newtoniana acerca del espacio, pero en este texto Kant no identifica esta realidad propia del espacio con Dios. En los últimos párrafos del opúsculo, Kant sostiene, como conclusión, que el espacio absoluto "es un concepto fundamental (Grundbegriff) que hace posible a toda sensación externa" (Ak. 2:383; traducción mía). En mi opinión, esto indica que, en el mejor de los casos, no es posible asegurar con exactitud si Kant mantenía una posición substancialista como la de Newton (en Ak. 17:334, reflexión atribuible al periodo de 1766-8, Kant sostiene que la relación (respectus) es algo positivo que puede ser pensado fuera de la sustancia y el accidente. Cf. Placencia 2007, pp. 71-2, nota 105). Respecto de (ii), me parece que en la afirmación del opúsculo 1968 de que el espacio hace posible toda sensación externa está el germen desde donde puede ser pensado que el espacio es una forma de la sensibilidad. Como sostiene Hintikka, todo lo que tendrá que hacer Kant respecto de la doctrina de Newton será cambiar a Dios por el hombre (Hintikka 1974, pp. 128-9). 
decisivo en orden al planteamiento más acabado de la siguiente cuestión, a saber, la posibilidad de que una representación en general (tanto sensible como intelectual) refiera a un objeto del mundo. A mi modo de ver, el solo hecho de que una representación cualquiera refiera a objetos del mundo implica tomar conciencia de una cierta pasividad de nuestro ánimo, en cuanto que somos afectados por objetos (receptividad), y de una cierta actividad por medio de la cual producimos una representación (a priori o a posteriori) que refiere a un objeto. Pero esta actividad es todavía más relevante desde el punto de vista de su relación con objetos, cuando se afirma que el hombre posee ciertas representaciones producidas a priori, es decir, que existen independientemente de la experiencia sensible en que se aplican.

Ya sea que esta referencia se considere desde el punto de vista de la sensibilidad, como una facultad (Vermögen) del conocimiento sensible, ya sea que se tome en consideración al entendimiento, como una facultad de conocimiento intelectual independiente de la sensibilidad pero aplicada al mundo a través de esta, la combinación de actividad y receptividad que conforma al ser humano en cuanto a su modo de conocer se proyecta como el problema de la unidad que debe existir entre las facultades de conocimiento en orden a conformar las condiciones de posibilidad de un acceso cognoscitivo a las cosas.

En mi opinión, el avance desde el estadio precrítico hacia la $\mathrm{KrV}$ debe ser comprendido precisamente como la toma de conciencia del problema recién mencionado; o bien, lo que es lo mismo, como la comprensión de que la justificación para que una representación pueda referir a un objeto radica en explicar cómo se relacionan el entendimiento y la sensibilidad en la conformación del conocimiento. Volveré sobre este punto más adelante.

\section{El dualismo entre sensibilidad y entendimiento}

La distinción precisa de estos dos poderes del ánimo (Gemüt), y a la vez la más cercana al planteamiento de la $K r V$, tiene su génesis en la Dissertatio, donde Kant establece por vez primera los argumentos para mostrar la idealidad de la sensibilidad, es decir, para mostrar que los datos empíricos suponen, ya en su condición de datos sensibles, una cierta forma que posibilita que ellos se presenten como tales. De este modo, la posibilidad de todo objeto empírico está condicionada por la existencia de una estructuración subjetiva formal independiente de la experiencia misma, esto es, una forma anímica que ponga a los datos sensibles (las sensaciones) dentro de un horizonte de perceptibilidad común, a partir de la cual sea posible, en primer lugar, la relación entre estos mismos datos y, a través de ella, también su existencia.

La exposición de los argumentos de la Dissertatio, que prueban la idealidad de la sensibilidad, será repetida, con algunas modificaciones, once años más tarde en la primera edición de la $\mathrm{KrV}$, y luego seis años después, en su segunda edición. Esto confirma que Kant, ya en 1770, estaba convencido de su doctrina de la idealidad de la sensibilidad, y que sigue convencido de ella incluso en las dos redacciones de su $\mathrm{KrV}$, al menos en cuanto a los argumentos que prueban su existencia a priori. Esto no 
quiere decir, empero, como aclara Falkenstein, que los argumentos de la Dissertatio sean simplemente repetidos en la $K r V$ sin una función distinta a la que cumplían en el texto de 1770. A pesar de que una lectura superficial indique una similitud entre los argumentos para el espacio y tiempo en una y otra obra, el trasfondo de la $\mathrm{KrV}$ reformula radicalmente la función de la sensibilidad pura en la estructura a priori del conocimiento de objetos ${ }^{6}$.

Un par de años antes de la Dissertatio, en el opúsculo de 1768, titulado Von dem ersten Grunde des Unterschiedes der Gegenden im Raume ('Sobre el fundamento primero de la diferencia de las orientaciones ${ }^{7}$ en el espacio'; en adelante Sobre el fundamento), Kant desarrolla el llamado 'argumento de las contrapartidas incongruentes' para mostrar cómo en dos figuras corpóreas con características absolutamente iguales y semejantes, pero no congruentes (como la mano izquierda y la mano derecha), hay una diferencia que supone de antemano la noción de espacio para poder distinguirlas. En términos generales, el argumento sostiene que la determinación de dos formas corporales que son completamente iguales y semejantes entre sí (einander gleich und änhlich) no depende exclusivamente de la relación y posición de sus partes entre sí $^{8}$. Como sostiene Kant, dos figuras dibujadas en un plano, que sean iguales y semejantes, coincidirán en cuanto a sus límites ${ }^{9}$. Pero esto no sucede cuando se agrega una tercera dimensión y se comparan cuerpos geométricos. En este caso, aunque haya dos cuerpos iguales y semejantes, todavía será posible una diferencia que emerge de la referencia de las partes que componen estos cuerpos a un todo espacial. Un cuerpo será completamente semejante (völlig ähnlich) a otro cuando la figura (Figur) de uno sea semejante a la figura de otro. Y será completamente igual (ganz gleich) a otro cuando ambos cuerpos tengan la misma magnitud de la extensión (Grösse der Ausdehnung) ${ }^{10}$. De este modo, si dos cuerpos son iguales y semejantes en el sentido recién descrito (como sucede en el caso de las manos humanas), pero aún diferenciables, será necesario buscar otro criterio de distinción. Según Kant, la orientación (Gegend) en el espacio es este

$6 \quad$ Cf. L. Falkenstein 1995, 58-71. Falkenstein reinterpreta en estos pasajes los argumentos del espacio y del tiempo a la luz de la afirmación de Kant de que nuestras intuiciones sin conceptos son ciegas (cf. A51/B75). Esta ceguera (Blindness) indica una relación inseparable entre la sensibilidad y el entendimiento. Además, cf. Torretti 2004, pp. 114-5.

7 Si bien en general puede entenderse por el término alemán Gegend lo que uno entiende en castellano por región, sigo acá la traducción por orientación, que está tomada de L. Placencia (2007, 25-26, n. 13). Véase esta misma nota para una discusión del problema que presenta la palabra utilizada por Kant.

Cf. Ak. 2: 381.

Cf. Ak. 2: 381 .

10 En De Analysi Situs, Lebniz establece una diferencia parecida entre cosas iguales (aequalia) y cosas similares o semejantes (similia) (GM 5: 153). En particular, la figura posee, además de la cantidad o magnitud (quantitas o magnitudo), la cualidad o forma (qualitas oforma) (GM 5: 179). 
criterio, que se constituye como una diferencia interna (ein innerer Unterschied) entre estos dos cuerpos ${ }^{11}$.

Ciertamente, como sostiene Kant, si el espacio es entendido como la relación externa de las partes de la materia, no podría haber diferencia entre un cuerpo y su contrapartida, pues en ellos las relaciones de sus partes son exactamente las mismas ${ }^{12}$. Si esto es así, los cuerpos serían indeterminados respecto de su orientación (Gegend). En el caso de la mano izquierda o derecha, esto significaría que su "ser derecha" o "ser izquierda" no sería relevante para determinar estas figuras corporales, lo cual es imposible ${ }^{13}$. Incluso si una mano fuese el único objeto existente en el mundo, ella tendría que ser izquierda o derecha ${ }^{14}$.

El punto central del argumento es que estos cuerpos solo se distinguen por su orientación en el espacio. Pero la captación de una orientación no depende del lugar de las partes de un cuerpo, sino de la referencia de estas a un todo espacial ${ }^{15}$. Y en este punto, precisamente, el espacio tiene prioridad ontológica respecto de los objetos que se encuentran en él, en cuanto que aquella totalidad espacial presupuesta determina el modo de ser de un objeto sensible en general, la cual no puede ser conocida ya mediante notas inteligibles, sino que solo puede ser intuida. Ello indica que el espacio, considerado por sí mismo, posee una entidad diferente al de la realidad intelectual. Sin embargo, en este opúsculo, Kant no desarrolla hasta sus últimas consecuencias esta idea, aunque ya está presente acá el germen, a partir del cual es posible establecer una distinción en origen y contenido (Ursprung und Inhalt) de dos facultades de conocimiento ${ }^{16}$.

En la Dissertatio, con todo, Kant tampoco concibió la radicalidad de esta doctrina de la sensibilidad pura, tal como ella es expuesta en la $\mathrm{Kr} V$, en cuanto a su conexión con el entendimiento. Antes bien, Kant presenta en 1770 un dualismo tajante entre las dos facultades de conocimiento. Se ve forzado a distinguir, aparentemente de un modo irreconciliable, las facultades del sujeto cognoscente en virtud de los principios del conocimiento que descubre en la misma actividad cognoscitiva. En el opúsculo de 1768, ya había entrevisto la posibilidad de un ámbito perteneciente exclusivamente a la sensibilidad con independencia de lo intelectual. Pero en 1770 , sostiene ya que hay dos facultades de conocimiento, a las que les corresponden principios de conocimiento totalmente distintos. Así, la sensibilidad posibilita la aparición de un objeto sensible, esto es, las apariencias (apparentia), porque posee principios que le pertenecen exclusivamente a ella, a saber, el espacio y el tiempo como formas de tal apariencia, que permiten la conformación de la materia sensible (sensatio). Y a su vez, el entendimiento posibilita

Cf. Ak. 2: 382.

Cf. Ak. 2: 383. Además, Torretti 2004, pp. 112-5 y Torretti 1992, pp. 17-24.

Cf. Ak. 2: 382-3.

Cf. Van Cleve 2003, p. 231.

Cf. Ak 2: 381-3.

Cf. $K r V$, A44/B61. 
la aprehensión de ciertos conceptos, que surgen por la naturaleza misma del intelecto y que, por lo tanto, en absoluto son abstraídos del ámbito sensible ${ }^{17}$.

Ciertamente, ambas facultades deben ser distinguidas de una manera rigurosa en virtud de los principios específicos que posee cada una de ellas. Ni el objeto propio de la sensibilidad podría ser conformado por los principios intelectuales ni el objeto propio del entendimiento podría corresponder a los principios del conocimiento sensible ${ }^{18}$. De ahí también, que Kant establezca aquí la vieja distinción entre Phaenomena y Noumena para referirse a los objetos propios de cada facultad, cuya significación tomará un rumbo diferente en el capítulo homónimo de la $\mathrm{Kr}^{19}$.

A pesar de esta dualidad de las facultades, Kant no niega en la Dissertatio que haya una interacción efectiva entre ambas. En efecto, dentro del conocimiento sensible, distingue entre apariencias (apparentia) y experiencia (experientia): la primera consiste en meras percepciones que preceden al uso lógico (usus logicus) del entendimiento, en tanto la experiencia se constituye por las mismas apariencias, pero relacionadas según las leyes lógicas del entendimiento. Pero esta intervención de la facultad intelectual en el ámbito sensible es meramente accidental, pues el entendimiento ordena objetos sensibles ya constituidos como tales previamente. En este sentido, Kant sostiene que, aunque exista una relación, no hay una confusión entre las facultades, pues los predicados 'sensible' e 'intelectual' solo se atribuyen a los objetos en virtud del origen que estos posean según los principios que los posibiliten ${ }^{20}$. Kant se da cuenta, por cierto, de que en el conocimiento de un objeto sensible no basta solamente la coordinación a priori espacio-temporal de los datos empíricos, sino que también es necesaria una

Cf. Ak 2:394, § 6 .

18 El siguiente pasaje es conflictivo: "la inteligencia (racionalidad) es la facultad del sujeto, por la que es capaz de representarse aquellas cosas que, en virtud de su propia constitución, no pueden caer en los sentidos mismos", Ak 2:392 (Intelligentia (rationalitas) est facultas subiecti, per quam, quae in sensus ipsius per qualitatem suam incurrere non possunt, repraesentare valet). Dependiendo de cómo se entiende el término per qualitatem suam, hay dos maneras de interpretar el pasaje: i) o bien es por la cualidad de las cosas que ellas no pueden caer en los sentidos, y así habría que atribuir aquella cualidad a los objetos inteligibles (cf. R. Torretti 2005, pp. 206-207 y D. Walford 2003, p. 384, quienes traducen de este modo); ii) o bien es por la cualidad propia de la facultad del sujeto que las cosas que se representa no pueden caer en los sentidos, y así habría que atribuir tal cualidad al sujeto cognoscente (cf. la traducción de W. Weischedel 1977, § 3, en cuyo caso el término en discusión va entre comas: (...) quae in sensus ipsius, per qualitatem suam, incurrere (...)). Me parece que esta última traducción podría forzar la relación del entendimiento con sus objetos, al sugerir que estos dependen del modo de ser del entendimiento. En la Dissertatio, Kant todavía atribuye a los objetos inteligibles una existencia de por sí con independencia de la facultad intelectual del sujeto humano y está lejos siquiera de sugerir tal comprensión. En vistas de esto, sigo la primera interpretación. Agradezco los comentarios del corrector sobre este punto.

19 Cf. E. Cassirer 2001, pp. 96-97. 
subordinación lógica de tales datos. Pero a pesar de esto, en esta obra no explica de ningún modo cómo sea posible la conexión de dos facultades que están constituidas como tales previamente a esta referencia mutua. Dicho de otro modo, es claro que en la Dissertatio Kant se refiere a una aplicación del entendimiento sobre la sensibilidad, no obstante lo cual ambas facultades no pierden su independencia la una con respecto a la otra, y se mantiene por tanto una constitución dual del sujeto según las leyes del conocimiento descubiertas. Consiguientemente, en el análisis de Kant de 1770 sobre las dos facultades de conocimiento, se sostiene una separación constitutiva de ambas capacidades de conocimiento, en que la una no requiere a la otra para llegar a ser una facultad de conocimiento ${ }^{21}$.

Aquí reside, en mi opinión, una de las diferencias importantes entre el pensamiento kantiano de 1770 y el desarrollo crítico de principios de 1780. La $\mathrm{KrV}$, no afirma que la conexión entre sensibilidad y entendimiento en el conocimiento sensible sea meramente accidental, tal como sostiene Kant en la Dissertatio. Al contrario, según la $\mathrm{KrV}$, las fuentes del conocimiento solo llegan a estar constituidas como tales, a mi modo de ver, en virtud de la relación que poseen entre sí. Sensibilidad y entendimiento no existen por sí mismas, con independencia de su mutua referencia, porque su existencia depende de la relación misma en que ellas se encuentran; y consiguientemente, cada una no existe por separado, ni podría hacerlo tampoco. Esto es lo que quiero decir cuando me refiero a que entre ambas facultades hay una conexión esencial, pues lo que constituye el ser de cada una, en cuanto facultad, es su relación con la otra. Si esto es así, como creo que es el caso, entonces el dualismo de la Dissertatio no se mantiene en la $\mathrm{KrV}$.

Además, aunque en la Dissertatio Kant considere que hay una interacción entre la sensibilidad y el entendimiento, de ahí no se sigue la superación del dualismo, pues explicar cómo el entendimiento se aplica sobre la sensibilidad no es, en ningún caso, haber explicado la legitimidad de tal aplicación. Y por ello, no hay una real justificación de la intervención lógica de una facultad sobre otra.

El problema del dualismo es, por los argumentos precedentes, innegable. Pero con esto no pretendo desconsiderar la importancia y la novedad que exhibía la exposición de Kant acerca de la sensibilidad en esta época, principalmente porque su separación de las facultades obedecía a la misma estructura interna de las leyes de cada tipo de conocimiento. Además, siguiendo a Falkenstein, es interesante que Kant apele, nuevamente, a la antigua distinción dual para la solución del problema del conocimiento. Los filósofos modernos, en opinión de este autor, habían dejado a un lado esta división, tratando de dar cuenta del problema bajo la consideración de una

21 Cf. L. Falkestein 1995, pp. 52-54. Véase también la interesante interpretación de Cassirer respecto del problema del dualismo en Kant y de cómo este se conecta con la problemática general de la $K r V$ : "el dato decisivo [para la consideración de dos mundos] -algo de lo cual Kant se volvió completamente consciente estudiando a Leibniz-radica en la distinta proveniencia (Herkunft) y en el distinto tipo de validez (Geltungsart) de los principios de nuestro conocimiento", E. Cassirer 2001, p. 97. Traducción mía. 
sola facultad. Así, por ejemplo, aun cuando Leibniz diferencie entre sensibilidad y entendimiento, tal separación es solo gradual, de modo que la sensibilidad no es otra cosa que un entendimiento confuso ${ }^{22}$.

Sin embargo, Kant se oponía fuertemente a esta idea de una facultad única, porque llevaba consigo dificultades importantes. Solo para poner un ejemplo de esta problemática, sin querer extenderme demasiado sobre esto, considérese la posición que debía tener la matemática dentro del conocimiento racional. Si se afirma que los principios de la geometría, por ejemplo, descansan en el mundo de la experiencia, se puede explicar cómo es posible su aplicación en el mundo empírico a costa de perder la universalidad y apodicticidad que la matemática ha arrogado para sí desde siempre. Si, por otro lado, se sostiene que sus principios son completamente universales y necesarios, se vuelve difícil explicar su completa aplicabilidad al mundo cambiante de los objetos sensibles $^{23}$. Sin embargo, debido a que el espacio y el tiempo son la forma a priori del conocimiento sensible, Kant considera que la geometría es el prototipo de este modo de conocimiento ${ }^{24}$, pues tomaba sus principios de una intuición pura.

Así, la idea de estos dos mundos separados, que en definitiva es la mantención de dos tipos diferentes de principios cognoscitivos, lograba conciliar un conocimiento que fuese particular y universal al mismo tiempo, sin que existiese una contradicción en ello. La matemática se imponía, entonces, como un claro ejemplo de esta armonía racional, pues tenía garantizada su independencia con respecto a la realidad empírica, al mismo tiempo que su total coincidencia con ella ${ }^{25}$. Pero esta conciliación no estaba aclarada aún en cuanto a su legitimidad.

\section{La cuestión metodológica: el carácter de lo intuitivo a priori}

Como hemos visto, la distinción entre la intencionalidad de ambas facultades está presente ya en la Dissertatio. Lo que Kant descubre con su doctrina de la sensibilidad pura es que hay una cierta relación con algo que es independiente de la experiencia (a priori), pero que a su vez no se relaciona con ese algo discursivamente, sino que tiene una relación inmediata, esto es, intuitiva. El tipo de referencia a este algo no se da, por tanto, de manera mediata a través de conceptos, sino de una manera espaciotemporalmente inmediata. Así, espacio y tiempo, como intuiciones puras, no son tomados de la experiencia como sucede con el conocimiento empírico, pero tampoco son intelectuales en el sentido de estar referidos al entendimiento.

22 Cf. tal alusión a Leibniz en Ak 2:394, § 7. Además, L. Falkenstein 1995, pp. 28-32 y H. Cohen 2006, pp. 81-83.

23 Cf. E. Cassirer 2001, pp. 97-103.

24 "Sensitivae cognitionis prototypo", cf. Ak 2:394-5. Además, cf. R. Torretti 2005, pp. 208212.

25 Cf. E. Cassirer 2001, p. 103 y L. Shabel 2010, pp. 113-115. 
El hecho de que Kant haya podido tener acceso a este nuevo ámbito del conocimiento, que se conforma por una intuición pura no intelectual, se debe, al menos en parte, a que utiliza un método que posee un carácter singular. Aunque no puedo entrar a detallar acá de una manera apropiada la cuestión metodológica involucrada en la descripción intuitiva de la sensibilidad pura, me gustaría exponer un par de observaciones, principalmente para intentar al menos una aproximación a la novedad filosófica que involucra la facultad sensible tal como Kant la entiende.

Según Ernst Cassirer, durante la época ilustrada en general existió un fuerte rechazo al método matemático deductivo para investigar la realidad, impuesto ya desde el pensamiento de Descartes, cuya reacción se manifestó en la imposición de otro método, distinto al deductivo, que consistía en el análisis de la realidad ${ }^{26}$. Creo plausible que Kant haya tomado muy en cuenta este nuevo principio de investigación, principalmente en cuanto a su doctrina de la sensibilidad se refiere. Si uno indaga, en efecto, el opúsculo de 1768, se puede dar cuenta de la enorme cantidad de análisis de casos particulares que realiza Kant para llegar a concluir, solo hacia el final de este texto, lo que comentamos más arriba, a saber, que el espacio tiene prioridad (ontológica) respecto de las cosas que se encuentran en él. Sin embargo, aunque sea verdad que en 1770 Kant se aleja de este tipo de análisis (y después en la $\mathrm{KrV}$ ), como se puede evidenciar en el modo de presentación de los argumentos de la Dissertatio, es significativo, a mi entender, que profundice acá una idea que fue extraída justamente a partir de un análisis caso por caso. Esto podría sugerir una adscripción de Kant a este nuevo tipo de metodología.

Por otro lado, en la Estética Trascendental, tanto de 1781 como de 1787, la $\mathrm{KrV}$ presenta sus argumentos para la demostración de la idealidad y de la estructura interna de la facultad sensible, haciendo una advertencia importante desde un punto de vista metodológico. Lo que hará tanto en relación con el espacio como en relación con el tiempo es una exposición (Erörterung; expositio), metafísica y trascendental, de estos conceptos. Kant define 'exposición' como una representación clara de aquello que pertenece a un concepto, aunque ella no sea exhaustiva ${ }^{27}$, esto es, aunque dicha representación no exponga todas las notas que le correspondan según su naturaleza a priori. Antes bien, esta exposición volverá explícito aquello que le corresponde a los conceptos, dejando abierta la posibilidad de una caracterización más completa de él.

La demostración del ser ideal del espacio y del tiempo no consiste, por lo tanto, en una deducción lógica ${ }^{28}$, sino, al menos en una primera instancia, en un análisis a partir del cual puede afirmarse que estos poseen un ser sensible, pero a priori (no

26 Para un desarrollo un tanto más extenso de esta idea, cf. E. Cassirer 1998, pp. 67-83. Sobre este cambio de método en la filosofía de Kant, cf. también R. Torretti 2005, pp. 157-161.

27 Cf. $K r V$, A23/B38.

28 Nótese que Kant reserva la palabra 'deducción' (Deduktion) para la demostración de los conceptos puros del entendimiento. No obstante, debe tomarse en cuenta que esta deducción refiere a la legitimidad (quid juris) del uso de conceptos puros en la experiencia. Cf. $K r V$, A84/B116 - A85/B118; además, cf. M. Heidegger 1991, p. 78 (§17). 
empírico). Ni la Dissertatio ni la $K r V$ presentan, ciertamente, un análisis empírico de casos particulares, pero ahondan la conclusión de Sobre el fundamento acerca de la consistencia del espacio y del tiempo en cuanto que son intuiciones puras y principios de conocimiento. Y en esta exposición, manifiestan una herencia metodológica nueva ${ }^{29}$.

En efecto, la diferencia entre sensibilidad y entendimiento se funda en el hecho de que a la primera pertenecen intuiciones, esto es, una referencia directa e inmediata al objeto, en tanto a la segunda pertenecen conceptos, es decir, una referencia indirecta y mediata al objeto ${ }^{30}$. En términos kantianos, esto quiere decir lo siguiente: que las partes contenidas en el concepto caen bajo él, es decir, el concepto está fuera de las partes que lo constituyen. Por ejemplo, el concepto 'hombre' con respecto a sus instanciaciones particulares. Se dice que tal concepto está fuera de las partes (sus instanciaciones), porque es una abstracción, esto es, cada una de sus partes no contiene totalmente lo que contiene el concepto. La instanciación del concepto posee más notas que lo que significa el concepto en general. Así, por ejemplo, las características de un hombre particular no se agotan simplemente en su humanidad, pues refieren a rasgos que lo particularizan como ese hombre. En este sentido, lo que este hombre sea no se contiene completamente en el concepto 'hombre'; y precisamente por ello, se dice que el concepto es una abstracción y que está fuera de sus partes.

En cambio, Kant descubre un tipo de relación radicalmente diferente entre el espacio y el tiempo como totalidades y como partes, pues cada una de estas contiene absolutamente lo que contiene el espacio o el tiempo como totalidad. El ser de una parte del espacio es, pues, igual al ser del espacio considerado como totalidad, porque muchos espacios y muchos tiempos pertenecen todos a un único espacio y a un único tiempo $^{31}$. Y precisamente por ello, se afirma que las partes del espacio están en el espacio, no más allá de él y que el espacio en general comprende en sí, no bajo sí, a sus partes que lo constituyen como tal.

De esta forma, tanto las partes de un concepto como las partes de una intuición contienen la totalidad de su concepto o intuición general, pero de manera diferente, porque en el primer caso, las partes caen bajo el concepto, es decir, las partes no son todo lo que es el concepto general, y en ese sentido están más allá del concepto. Pero en el segundo caso, las partes están en la intuición, es decir, las partes son todo lo que es la intuición general, de modo que esta comprende en sí a sus partes ${ }^{32}$.

La formulación de este argumento, aunque no demuestra directamente la aprioridad de las formas espacio temporales, muestra su intuitibilidad (Anschaulichkeit), y con ello su pertenencia a otra facultad de representación (Vorstellungsfähigkeit), a saber, la 
sensibilidad como facultad de conocimiento ${ }^{33}$. El argumento muestra, pues, que espacio y tiempo constituyen el aspecto subjetivo de la sensibilidad ${ }^{34}$. En conformidad con el argumento de A25/B39 de la $K r V^{35}$, Kant comprende que esta relación del espacio y del tiempo con sus partes implica una unidad común en ellas, cuya multiplicidad solo le adviene en la medida en que haya una limitación de esta totalidad unitaria. Nuestros diversos conceptos de espacios o representaciones espaciales en general solo tienen su posibilidad en cuanto espaciales debido a una unidad previa en la que estos conceptos se basan ${ }^{36}$. Y esta idea de que el espacio como intuición sea previo, no quiere decir que sea anterior en el orden de la generación de estos conceptos, sino en el sentido en que es anterior el fundamento respecto de lo fundamentado. Por esta misma razón, Kant no asume una generación psicológica de los conceptos de espacio y tiempo, como si primero (en el orden de la generación) adquiriésemos sensaciones aisladas, y luego sobre ellas se impusieran las formas de espacio y tiempo ${ }^{37}$. Antes bien, el objeto mismo representado es aquello en el cual puede deslindarse su fundamento sensible, de tal modo que la particularización de ese espacio y ese tiempo no es nada más que una limitación o restricción de una unidad más fundamental, que yace como condición de posibilidad de esa misma concretización.

Por ello, esta unidad fundamental sensible que Kant descubre y nombra como intuición, a saber, la forma de la sensibilidad, expresa una clara diferencia entre una caracterización puramente intelectual y una caracterización estrictamente sensible. El ser sensible de los principios del conocimiento, que constituye la causa de otra facultad de conocer, se funda en el ser particular puro (como opuesto al ser general) de las formas que la constituyen, es decir, en el hecho de que ellas sean intuiciones, y no conceptos.

33 Cf. $K r V, \mathrm{~B} 150$.

34 Cf. R. Brandt 1998, p. 90.

35 Este es el argumento: "Él [el espacio] es esencialmente uno; lo múltiple en él, y por lo tanto también en el concepto común de los espacios en general, consiste solamente en limitaciones. De aquí se sigue que, en atención a ellas, yace como fundamento una intuición a priori (que no es empírica) de todos los conceptos del espacio (von demselben)". Traducción mía.

36 Esta unidad previa que Kant afirma estar presente en el espacio sin haber expuesto aún el principio supremo de toda experiencia en la 'Deducción de los conceptos puros', a saber, la apercepción trascendental', podría entrar en conflicto con la unidad del sujeto que requiere la experiencia. Sostengo que esta unidad del espacio y la unidad de los conceptos es exactamente la misma, y manifiesta, al fin y al cabo, la superación del dualismo presente en la Dissertatio. Cf. KrV, B160-161, nota; además, B. Longuenesse 1998, pp. 211-227.

37 Para una consideración sobre esta interpretación psicológica, cf. H. J. Paton 1961, pp. 137- 143. Por su parte H. Cohen, en Kants Theorie der Erfahrung, discute constantemente contra interpretaciones psicologistas; cf. H. Cohen 2006, pp. 105-109, por ejemplo. 


\section{El origen del problema crítico. Actividad y receptividad}

Ahora bien, creo que debe tenerse especial cuidado por lo que se entiende bajo los conceptos de espacio y tiempo, incluso en el periodo precrítico de la Dissertatio. Para su comprensión, me parece fundamental hacer dos precisiones al respecto. Por un lado, (i) el espacio y el tiempo puros, en cuanto que son condiciones de objetos sensibles, no son ellos mismos otros objetos ya constituidos con anterioridad a lo que condicionan, sino que son lo que relaciona a los objetos espaciotemporales, y en este sentido, son relación pura. Pero al mismo tiempo, como condiciones relacionantes, no existen al margen de lo que relacionan, esto es, no existen sin algo que relacionar (aún cuando sea un objeto posible), pues su particular modo de ser está referido necesariamente a objetos sensibles. Esto lleva en sí la idea de que espacio y tiempo puedan constituirse como la sola posibilidad de que los objetos se constituyan de un modo espaciotemporal, cuya activación como principios efectivos necesita de la sensación (Empfindung).

Por otro lado, (ii) tales condiciones de posibilidad no se adhieren a los objetos sensibles de una manera accidental, como si estos objetos pudiesen existir sin el espacio y el tiempo. Lo que a mi modo de ver Kant entiende con respecto a su doctrina de la sensibilidad, tanto en la Dissertatio como en la $\mathrm{KrV}$, es que la forma de la sensibilidad le da su condición de ser sensible a un objeto. Esto quiere decir que todo aquello que caiga bajo las formas de espacio y tiempo debe ser sensible, porque la materia de un objeto de conocimiento (sensación) es siempre materia para un sujeto, es decir, materia que debe caer bajo las formas anímicas de espacio y tiempo para que pueda presentarse como algo conocido. Por esta razón, sostengo que esta materia no se presenta, por decirlo de alguna manera, en bruto, sino que solo llega a ser "materia para un sujeto", es decir, conocimiento, en la misma medida en que ella se conforme a lo que el sujeto pone activamente, esto es, la forma espaciotemporal. Y en este sentido, el tipo de ser que es la sensación no solo es un ser sensible por causa de la forma anímica de la sensibilidad, sino que este ser, la sensación, es el único que se le puede presentar a un sujeto con esta configuración formal. Dicho de otro modo, para el sujeto no existe una materia oculta o detrás del fenómeno (Erscheinung), pues la única materia a la que puede acceder es a aquella que se presenta bajo la forma espaciotemporal.

Sin embargo, ya se considere esta sola materia, ya esta forma subjetiva espaciotemporal, no debe perderse de vista que ambas llegan a manifestarse como lo que son exclusivamente en su mutua referencia. Dicho de otro modo, no es posible volvernos conscientes de la materia del conocimiento (sensación) a no ser porque primero la hemos percibido bajo una forma espacio temporal ${ }^{38}$. Y precisamente, qué sea esta materia en sí misma, con independencia de su referencia al modo de ser percibida, es imposible de ser conocido. Por esta razón, el espacio puro no es el arriba o el abajo, ni el tiempo puro es el pasado o el presente, sino más bien la conciencia de la receptividad 
de impresiones de cosas conforme a ciertas relaciones entre sí $^{39}$. Espacio y tiempo son leyes ínsitas al sujeto, conforme a las cuales la sensación puede ser coordinada de un modo determinado. Por ello, la particularidad o especificidad de esta ley sensible en una materia no le viene en virtud de ella misma como ley, sino en virtud de la materia en la que ella se da (sensación). De ahí que exista este tiempo o este espacio, porque un objeto sensible representa el modo particular en que la ley se especifica, es decir, el modo concreto en que ella se activa como ley.

Si bien el objeto sensible toma su "ser sensible" de las formas de espacio y tiempo, me abstengo de comentar por ahora el origen de su "ser objeto", principalmente porque la conciencia de esto último solo se realiza en la $K r V$. La Dissertatio no tenía presente el problema de cómo sea posible que algo llegue a ser un objeto de conocimiento. Pero lo que sí visualiza, con toda claridad, es la necesidad de una coordinación a priori de las sensaciones, esto es, de un orden determinado sensible que no proviene desde los mismos datos coordinados, pero que tampoco existe más allá de la materia misma, sino que es un principio de determinación interno al sujeto ${ }^{40}$.

Ahora bien, me parece que esta distinción entre la forma y la materia del ser sensible descrita en la Dissertatio trae consigo un planteamiento de orden más general, que no aparece planteado en este mismo texto. En efecto, la materia del conocimiento es necesaria solo para un ser que no es capaz de crear su propio objeto de conocimiento, sino que requiere que este le sea dado, al menos en parte. La materia es precisamente aquello que no es puesto por el sujeto. Por esto, creo que es significativo resaltar el hecho de cómo el sujeto se constituye, a la vez, como algo activo y receptivo respecto de un objeto sensible, porque aun cuando tenga la posibilidad de ser afectado por objetos, su receptividad no es pura pasividad, sino que tiene una cierta estructura (activamente) determinante. La forma de la sensibilidad funda la posibilidad de ser afectado, pero, por esta misma razón, este tipo de afección no es totalmente pasivo. Por esto, puede resultar paradójica la formulación de los principios sensibles como 'principios de la

Cf. AK. 17:638-9. Además, Torretti 2004, pp. 115-117.

40 Sostiene Kant en la Dissertatio: "Sin embargo, en la representación del sentido se encuentra primeramente aquello que puede denominarse materia, es decir sensación, pero además aquello que puede llamarse forma, es decir configuración (species) de lo sensible, que se manifiesta en cuanto aquello (varia) que afecta a los sentidos es coordinado mutuamente de cierto modo por una ley natural del espíritu (animi). Además, como la sensación, que constituye la materia de la representación sensible, revelando por cierto la presencia de algo sensible, depende en cuanto a su constitución (quoad qualitatem) de la naturaleza del sujeto, en la medida en que él es modificable por este objeto, así también la forma de esta precisa representación expresa una cierta referencia o una relación de lo sentido, pero que no es propiamente un bosquejo o un esquema del objeto, sino nada menos que una determinada ley ínsita a la capacidad de conocimiento (menti) de coordinar lo sentido que proviene de la presencia del objeto", Ak 2:392-3, §4 (traducción mía). Para la palabra menti, sigo la traducción de W. Weischedel (1977) para mens: 'Erkenntniskraft'; cf. 31. H. Cohen reinterpreta la idea de 'ser innato' en el sentido kantiano de 'ser a priori', cf. H. Cohen 2006, pp. 93-94 y 102-105. 
receptividad' ${ }^{41}$, según la cual espacio y tiempo son las condiciones de la afección o de la receptividad del sujeto, ya que, en cuanto que constituyen un poder (Vermögen) del ánimo, representan una cierta actividad, a saber, la de ser principios activos respecto de la constitución de la posibilidad del conocimiento.

En la Dissertatio, Kant ya percibe que hay en la facultad sensible una cierta actividad que está directamente relacionada con la función que le corresponde ejecutar, a saber, la de ser la forma de lo sensible de objetos empíricos. En un comienzo, pareciera que se asume, sin ninguna cautela, que el modo de recepción tiene que ser activamente un principio, sin tomar conciencia de la pregunta por la unidad de los principios del conocimiento. En efecto, el problema del dualismo aun subsiste en dos facultades separadas de modo radical, cuya manera de constituirse como principios del conocimiento (aislados) se asume como algo perteneciente exclusivamente a cada facultad: el entendimiento posee sus propios conceptos puros, así como la sensibilidad se forma en base a sus intuiciones puras. Pero me parece que, bajo esta concepción de las facultades del conocimiento, la idea de principio (principium o Grund), por medio de la cual se caracteriza las formas de la sensibilidad, no se lleva hasta sus últimas consecuencias, pues la independencia entre el entendimiento y la sensibilidad de la Dissertatio no permite mostrar aún el profundo arraigo que posee la unidad de la intuición a priori (que es sensible-receptiva) en la unidad del concepto a priori (que es intelectual-activa). Sostengo que en la medida en que sea posible mostrar este arraigo, será posible, al mismo tiempo, entender por qué la sensibilidad es descrita como una facultad activa. Dicho de otro modo, la actividad que posee la sensibilidad en su aspecto formal está directamente conectada con la actividad de la facultad conceptual.

Por esta razón, creo que el avance desde la Dissertatio hacia la $\mathrm{KrV}$ está directamente relacionado con la conciencia que se tenga de la noción de principio en la fundamentación del conocimiento. Desde el punto de vista dualista que mantiene la Dissertatio, el sujeto humano queda como partido en dos ámbitos que no pueden ser relacionados, a no ser accidentalmente (que, en definitiva, es lo mismo que no tener relación alguna). Esto significa que no se puede justificar el modo unitario de surgimiento de ambas facultades y, por lo tanto, no se puede mostrar tampoco cómo es que una apariencia sensible (representación), que denota un objeto sensible, pueda ser descrita precisamente en cuanto a su denotación, esto es, en cuanto a su referencia objetiva. $\mathrm{O}$ bien, lo que es lo mismo, no se puede justificar de qué modo es posible que un mero estado subjetivo del conocimiento, como una representación sensible (a partir de la cual se puede hablar de un objeto sensible), pueda ser señalado como perteneciente a un objeto de conocimiento, y por lo tanto a una fijación o necesidad que no radica en, y es completamente ajeno a, este estado subjetivo como tal.

41 Cf. Ak 2:396-7. Aquí Kant utiliza la palabra latina principium, que Weischedel traduce por Grund. Además, en la $K r V$ Kant se refiere al espacio y al tiempo como 'condiciones de la receptividad' (cf. $K r V$, A77/B102), como 'fundamentos' (cf. KrV, A23/B38 y A30/B46) y como 'principios' (cf. $K r V$, A21/B36, B40 y B51). 
La $K r V$ también se refiere múltiples veces a la sensibilidad como receptividad o pasividad, pero en ella existe más conciencia respecto de su rol principial en el conocimiento. Creo que si se profundiza en la idea kantiana de 'principio del conocimiento sensible', como en mi opinión hace efectivamente la $K r V$, entonces será posible desentrañar el problema del dualismo, en la misma medida en que se vuelva a pensar la idea de una facultad receptiva. Este último concepto entraña una combinación de actividad y receptividad, cuyo origen se desconoce y simplemente se asume como un hecho en la época de 1770.

La pregunta que uno podría hacer, entonces, es cómo fue que Kant llegó a sostener en la $K r V$ que la unidad de la intuición correspondía a la unidad conceptual, solucionando así el problema del dualismo ${ }^{42}$. Creo que el paso decisivo para el tránsito entre la Dissertatio y la $\mathrm{KrV}$ para la resolución del dualismo está dado en la carta dirigida a Marcus Herz el 21 de febrero de 1772. Esta carta muestra cuál es la relación entre la afirmación de que la sensibilidad sea una facultad receptiva, ya presente en la Dissertatio bajo la idea de que espacio y tiempo son el aspecto formal de un conocimiento sensorial, por una parte, y la necesidad de que nuestras representaciones sean objetivas de modo tal que sea posible hablar de objetos sensibles, por otra.

En esta carta, Kant se da perfectamente cuenta de que la dificultad principal de su concepción dualista de la realidad, tal como la entiende en la Dissertatio, es el desconocimiento del fundamento de la referencia de nuestras representaciones a objetos. Para explicar este problema, primero deben atenderse los dos modos de entender esta referencia: en primer lugar, $(i)$ la representación puede componerse pasivamente de la manera en que el sujeto es afectado por el objeto, de modo que la relación entre objeto y representación se da en términos de causa y efecto. La representación sería, en este caso, solo un efecto del objeto. Y en segundo lugar, (ii) si lo que llamamos 'representación' fuese activa y produjese su objeto, de tal modo que este último sería solo el efecto de aquella representación. En este caso, la representación sería la causa del objeto. Kant piensa acá, por ejemplo, en un entendimiento divino que crea su propio objeto, un intellectus archetypus cuya intuición es el mismo fundamento de las cosas y cuya relación con objetos es, por ello, completamente inmediata y suficiente. Sin embargo, dentro de (ii), también considera un entendimiento pasivo que nada tiene que ver con la constitución del objeto mismo, un intellectus ectypus cuyo proceder lógico deriva sus objetos (lógicos) de las representaciones sensibles que llegan sobre él ${ }^{43}$.

No obstante esta división, el problema es que el entendimiento humano ni es puramente pasivo en el sentido de $(i)$, ni es puramente activo en el sentido de (ii), sino que es una mezcla de actividad y receptividad. Al respecto, dice Kant:

$42 \quad$ Cf. nota 34.

43 Creo que Kant está pensando en el uso lógico (usus logicus) del entendimiento descrito en la Dissertatio. Cf. Ak 2:393, §5. 
Sin embargo, nuestro entendimiento, mediante sus representaciones, ni es la causa del objeto (salvo en el caso de los fines morales), ni el objeto es la causa de nuestras representaciones intelectuales en el sentido real (in sensu reali). Por lo tanto, los conceptos puros del entendimiento ${ }^{44}$ no tienen que ser abstraídos de las percepciones, ni tienen que expresar la recepción de representaciones a través de los sentidos; pero aunque ellos tengan que tener su origen en la naturaleza del alma, no son causados por el objeto ni provocan que el objeto mismo exista (Ak 10:130. Esta cita y la siguiente siguen la traducción inglesa de Arnulf Zweig ${ }^{45}$ ).

En relación, por un lado, con el problema del dualismo que arriba había planteado y, por otro, con la conciencia de que una capacidad receptiva como la sensibilidad también es, en cierto modo, activa, se podría decir que el foco del asunto se traslada en esta carta desde la consideración de los principios mismos de la sensibilidad (como activosreceptivos) hacia la misma actividad del entendimiento (como activa-receptiva). El problema que plantea Kant en esta carta se refiere a la pregunta por la posibilidad de que los conceptos propios del entendimiento (como el concepto de sustancia o causalidad) puedan decir algo acerca de las cosas del mundo, dado que estos no provienen de los objetos sensibles (percepciones) a los que supuestamente se adecuan. En efecto, estas representaciones intelectuales (conceptos) no provienen de la experiencia, pero a su vez tampoco pueden crear los objetos del mundo de la experiencia. Así, el entendimiento, que es la facultad que soporta esta clase de conceptos, se constituye como una mezcla de actividad y pasividad, que no se corresponde con ninguno de las dos clasificaciones de referencia a las que referí supra. En efecto, ¿cómo es posible que los conceptos del entendimiento se adecuen a un objeto del mundo, si ellos mismos no son la causa de este objeto (no lo producen, como sí hace el entendimiento divino) ni, a su vez, el sujeto ha sido afectado por él, ya que tales conceptos no han sido derivados de las representaciones sensibles?

Las representaciones intelectuales en sentido real (no en sentido lógico), según la Dissertatio, presentaban las cosas tal como ellas eran en sí mismas. Pero frente a este problema, permanece insoluble la cuestión de cómo sea posible que una representación en general pueda referirse a una cosa tal como ella es en sí misma sin ser afectada por ella ni sin que ella produzca tal cosa. Es decir, en este período de su pensamiento, pareciera que el real problema para Kant es explicar cómo un entendimiento finito, que no es completamente receptivo, debido a que posee representaciones en virtud de su propia actividad, ni completamente activo, debido a que no produce los objetos de estas representaciones, puede lograr una cierta conformidad con sus objetos de conocimiento. Este problema es trazado con toda su fuerza en el siguiente pasaje de la carta a Herz:

Kant no está pensando acá en los conceptos puros que en la $K r V$ llamará también, de un modo más técnico, categorías. 
Pero en el caso de las relaciones que envuelven cualidades -tanto el hecho de cómo mi entendimiento puede formar, completamente a priori, por sí mismo conceptos de cosas con los que los hechos debieran necesariamente adecuarse, como el hecho de cómo mi entendimiento puede formular principios reales que conciernen a la posibilidad de tales conceptos, con los cuales la experiencia tiene que estar en una exacta adecuación y que sin embargo son independientes de la experiencia- esta cuestión de cómo la facultad del entendimiento logra esta conformidad con las cosas mismas está aún abandonada en un estado de oscuridad (Ak 10:131).

Es interesante notar acá que si el entendimiento tiene una existencia por sí mismo, es decir, como una facultad con derecho propio, por decirlo así, entonces la resolución de la cuestión que Kant plantea en este pasaje involucra directamente la legitimidad de que exista el entendimiento como una facultad de conocimiento. En efecto, si los conceptos intelectuales (o representaciones intelectuales) no proveen un conocimiento acerca de las cosas del mundo, como se supone que debería ser el caso si representan a las cosas tal como son en sí, se llega a un mero escepticismo, pues en definitiva sería imposible conocer. Para solucionar este problema, es clave resolver de qué forma los conceptos del entendimiento pueden lograr una adecuación a los objetos sensibles que provee la sensibilidad.

Pero al mismo tiempo que se plantea este problema, se deberá interpretar la sensibilidad como facultad de conocimiento desde otro punto de vista, distinto del que tenía la Dissertatio. En efecto, el dualismo de este texto impide solucionar el problema recién descrito en la carta a Marcus Herz, pues supone que la unidad entre el entendimiento y la sensibilidad no es necesaria para la fundamentación del conocimiento. La necesidad de justificar el modo en que una representación refiere a su objeto en el mundo requiere explicar, necesariamente, cómo es posible que el entendimiento pueda relacionarse con la sensibilidad, que es la que provee representaciones empíricas, a pesar de que ambas facultades proveen diferentes tipos de conocimiento. Esto implica, pues, renovar el concepto de sensibilidad y comprenderlo precisamente como una capacidad receptiva que posee una estrecha relación con la actividad del entendimiento. Esta mutua determinación entre sensibilidad y entendimiento permitirá profundizar el concepto de principio que está involucrado en las formas de espacio y tiempo de la sensibilidad, de modo que sea posible presentar los datos de la sensibilidad como si poseyesen determinaciones propias del entendimiento, como es el caso de la permanencia de las sensaciones (sustancia). Evidentemente, esta explicación no se logra en la Estética Trascendental de la $\mathrm{Kr} V$, donde se presentan principalmente los argumentos a favor de un conocimiento intuitivo a priori de un modo muy similar a como fueron expuestos en la Dissertatio. El paso decisivo para la resolución de este doble problema, a saber, la comprensión de la sensibilidad como una facultad receptiva y la comprensión de cómo sea posible que una representación intelectual pueda referirse a un objeto sensible, será dado en la Deducción de los Conceptos Puros; e implicará, por cierto, una renovación de la noción de objeto para entender qué se quiere decir cuando se habla de un objeto sensible a partir de la sensibilidad. 
Hasta la carta dirigida a M. Herz, sin embargo, Kant no ha tomado conciencia de la radicalidad de estos problemas ni todos los matices involucrados en ellos. A pesar de que, como afirma Cassirer, todos los criterios de que habría de salir la $\mathrm{KrV}$ se hallan implícitos en el planteamiento de esta carta y de que, precisamente acá, ya se ha efectuado lo que Kant llamará más tarde la revolución del modo de pensar, el viraje copernicano del problema del conocimiento ${ }^{46}$, Kant no había percibido aún toda la extensión del problema ni su completa solución. Solo una muestra indirecta de ello es el hecho de que programaba dedicarle a estas investigaciones tres meses, como afirma en esta misma carta a Herz, aunque termina su labor recién nueve años después con la primera edición de la $\mathrm{KrV}$.

\section{Referencias bibliográficas}

KANT

Kants Werke, herausgegeben von der Königlich Preussischen Akademie der Wissenschaften. Berlin, 1968.

Kritik der reinen Vernunft, herausgegeben von J. Timmermann. Hamburg: Felix Meiner Verlag. 1998.

Schriften zur Metaphysik und Logik (1 und 2), Bände V und VI, herausgegeben von Wilhelm Weischedel. Frankfurt: Suhrkamp Verlag. 1977.

Correspondence, translated and edited by Arnulf Zweig. Cambridge: Cambridge University Press. 1999.

Theoretical philosophy 1755-1770, translated and edited by David Walford. Cambridge: Cambridge University Press. 2003.

LEIBNIZ

Matematische Schriften, herausgegeben von C. I. Gerhardt, Band V. Die matematischen Abhandlungen. Hildesheim und New York: Georg Olms Verlag.

Allison, H. (2004), Kant's Trascendental Idealism. New Haven: Yale University Press.

Brandt, R. (1998), Transzendentale Ästhetik, $\S ~ 1-3$; in Kritik der reinen Vernunft, herausgegeben von Georg Mohr und Marcus Willaschek, Abschnitt 4. Berlin: Akademie Verlag.

Cassirer, E. (2001), Kants Leben und Lehre. Gesammelte Werke, Band 8. Text und Anmerkungen bearbeitet von Tobias Berben. Hamburg: Felix Meiner Verlag. 1994 (1932), Filosofía de la Ilustración. Trad. Eugenio Ímaz. México: FCE. 
Cohen, H. (1871), Kants Theorie der Erfahrung. Saarbrücken: Dr. Müller e. K. und Lizenzgeber Verlag. 2006.

Falkenstein, L. (1995), Kant's Intuitionism. A commentary on the Trascendental Aesthetic. Toronto: University of Toronto Press.

Heidegger, M. (1991), Gesamtausgabe, Band 3: Kant und das Problem der Metaphysik. Frankfurt am Main. Vittorio Klostermann Verlag.

Hintikka, Jaakko (1974), Knowledge and the Known. Historical Perspectives in Epistemology. Boston: D. Reidel Publishing Company.

Longuenesse, B. (1998), Kant and the Capacity to Judge, translated by Charles T. Wolfe. Princeton and Oxford: Princeton University Press.

Molina, M. (2012), El surgimiento de la sensibilidad pura. Pamplona: Cuadernos de Anuario Filosófico.

Paton, H. J. (1961), Kant's Metaphysics of Expierence. A commentary on the first half of the Kritik der reinen Vernunft. 2 vols. London: George Allen \& Unwin Ltd. New York: The Macmillan Company.

Placencia, L. (2007), La ontología del espacio en Kant: Un estudio genéticosistemático sobre los fundamentos metodológicos y metafísicos de la teoría del espacio como intuición pura en la "Estética trascendental". Pamplona: Cuadernos de Anuario Filosófico.

Shabel, L. (2010), The Trascendental Aesthetic, in The Cambridge Companion to Kant's Critique of pure Reason, edited by Paul Guyer, chapter 4. New York: Cambridge University Press.

Torretti, R. (2005), Manuel Kant. Estudio sobre los fundamentos de la filosofía crítica. 3 tomos. Santiago de Chile: Ediciones Universidad Diego Portales (edición corregida).

(2004), Intuición Pura, en El sentimiento de lo humano en la ciencia, la filosofia y las artes. César Ojeda y Alejandro Ramírez, eds., Santiago de Chile: Editorial Universitaria.

(1992), La geometría en el pensamiento de Kant, en Variedad de la Razón. Ensayos sobre Kant. Carla Cordua y Roberto Torretti. Puerto Rico: Editorial de la Universidad de Puerto Rico.

Vaihinger, H. (1892), Commentar zu Kants Kritik der reinen Vernunft (Zweiter Band). Stuttgart, Berlin, Leipzig: Union Deutsche Verlagsgesellschaft.

Van Cleve, J. (1999), Problems from Kant. Oxford and New York: Oxford University Press. 